\title{
Surveillance of Mungbean Yellow Mosaic Virus (MYMV) Incidence and its Vector Population for Development of Yellow Mosaic Disease Forewarning
}

\author{
Meghashree Meti, Mallikarjun Kenganal*, D.S. Ashwathnarayana and Gururaj Sunkad
}

Department of Plant Pathology, College of Agriculture, Raichur, University of Agricultural

Sciences, Raichur 584104, Karnataka, India

*Corresponding author

\begin{abstract}
Keywords
MYMV, Whitefly population, SMW,

Correlation and

Regression.

Article Info

Accepted:

04 September 2017

Available Online:

10 October 2017 Wadagnal during kharif 2016. The results revealed that, the highest whitefly population observed during $26^{\text {th }}$ SMW at Kavalur and $29^{\text {th }}$ SMW at Raichur and Wadagnal locations during crop growth. The influence of weather parameters was studied by correlation and regression statistical analysis. The correlation analysis revealed that, maximum temperature was positively significantly correlated with whitefly population at Kavalur and Wadagnal villages ( 0.079 and 0.721 respectively) whereas minimum temperature was negatively correlated with whitefly population at Kavalur and Raichur (0.889 and 0.684 respectively). The per cent disease incidence showed negatively significant correlation with maximum temperature at Kavalur and Raichur ( 0.737 and 0.707 respectively). The rainfall was negatively correlated but non-significant with both whitefly population and per cent disease incidence at all the three locations. Correlation between whitefly population and disease incidence showed positive non-significant relation with each other in all the locations. The regression analysis revealed that, per cent contribution of weather factors on whitefly population and disease incidence at Kavalur, Raichur and Wadagnal was 98.3 percent $\left(R^{2}=0.983\right), 97.8$ per cent $\left(R^{2}=0.978\right)$ and 97 per cent $\left(R^{2}=0.970\right)$
\end{abstract}

\section{A B S T R A C T}

Surveillance of Mungbean Yellow Mosaic Virus (MYMV) on mungbean was undertaken to know the effect of weather factors on whitefly population (Bemisia tabaci Genn.) and MYMV incidence at three fixed locations viz., Kavalur, Raichur (UASR, Campus) and

\section{Introduction}

Mungbean (Vigna radiata (L.) Wilczek) is one of the thirteenth food legumes grown in India and third most important pulse crop after chickpea and pigeonpea. Mungbean is grown principally for its protein rich edible seeds which are used for consumption by cooking, fermenting, milling or sprouting. The crop is mainly cultivated during kharif season under rainfed conditions. Its cultivation has been hampered by many biotic and abiotic stresses. Among yellow mosaic virus disease of mungbean is considered as

serious threat, limiting the production and productivity. Mungbean yellow mosaic virus belongs to the family Geminiviridae (Geminate means twin particles) consisting of viruses with circular $(20 \times 30 \mathrm{~nm})$, singlestranded (ss) DNA genome (Hull, 2004). These viruses are transmitted from one plant to other plant through arthropod vectors with twin particles. Geminiviridae is taxonomically divided into four genera viz., Mastrevirus, Curtovirus, Topocuvirus and Begomovirus based on host range, genome organization and 
insect vector. The genus Begomovirus contains viruses that are transmitted by whitefly (Bemisia tabaci Genn.) infecting dicotyledonous plants like urdbean, mungbean and soybean (Haq et al., 2011).

The weather parameters play a vital role in survival and multiplication of white fly $(B$. tabaci) and influence the outbreak of MYMV in mungbean during crop season. Therefore, understanding of weather factors and their role in MYMV incidence is a prerequisite to provide base line information for developing disease forewarning system. However, studies in this regard and epidemiological aspects of MYMV are scanty in the North Eastern Karnataka region. Hence the studies were conducted to know the influence of weather parameters on whitefly population and MYMV incidence.

\section{Materials and Methods}

Surveillance of MYMV was undertaken during kharif, 2016 at three fixed locations of Koppal (Kavalur and Wadaganal) and Raichur (UASR, campus) districts. In each location, farmers field cultivating mungbean and nearby a functional weather observatory (within $1 \mathrm{~km}$ ) monitored by Karnataka State Natural Disaster Management Center (KSNDMC), Yelahanka, Bangalore were selected. The GPS readings of selected plots were noted and in each plot, yellow colour whitefly sticky traps (insect traps) of $33 \times 22$ $\mathrm{cm}$ size were placed at weekly interval beginning from the date of sowing $\left(23^{\text {rd }}\right.$ SMW) till harvest ( $33^{\text {rd }}$ SMW) of the crop to know the vector population at weekly intervals. The disease incidence was recorded at weekly interval (starting from first appearance of symptoms). The meteorological data at all the three locations was recorded at nearby observatory maintained by KSNDMC. The daily data collected from KSNDMC was computed to weekly interval and used for further correlation studies along with vector population recorded were done using SPSS 16.0 software. Statistical analysis of obtained data was studied through correlation to find the seasonal population dynamics of whitefly on mungbean with different weather parameters viz., temperature, Relative humidity, wind speed and rainfall. Relationship between whitefly, MYMV incidence and different meteorological variables were subjected to study using simple correlation and regression.

\section{Results and Discussion}

To find the association of whitefly population with weather parameters, data were pooled and correlation was worked out between whitefly population, per cent disease incidence and weather parameters. The results revealed that, disease incidence initially were zero percent at all three locations and incidence started from $26^{\text {th }} \mathrm{SMW}$. The highest whitefly population observed during $26^{\text {th }}$ SMW at Kavalur (Table 1) when maximum temperature of $29.71^{\circ} \mathrm{C}, \quad$ minimum temperature $22.59^{\circ} \mathrm{C}$, rainfall of $23.8 \mathrm{~mm}$, minimum relative humidity $(61.40 \%)$, maximum relative $(93.84 \%)$ and wind speed (2.29 $\mathrm{km} / \mathrm{hr})$. In case of Raichur and Wadagnal high whitefly population noticed at $29^{\text {th }}$ SMW (Table 5) (maximum temperature $34.01^{\circ} \mathrm{C}$, minimum temperature $26.00^{\circ} \mathrm{C}$, maximum relative humidity $94.81 \%$, minimum relative humidity $59.51 \%$ and wind speed $1.99 \mathrm{~km} / \mathrm{hr}$ at Raichur) and (Table 3) (maximum temperature $30.29^{\circ} \mathrm{C}$, minimum temperature $21.86^{\circ} \mathrm{C}$, maximum relative humidity $88.57 \%$, minimum relative humidity $54.86 \%$ and wind speed $5.04 \mathrm{~km} / \mathrm{hr}$ at Wadagnal) in each locations during crop growth. The least number of whitefly/trap was observed during high rainfall in all locations which indicates more the rainfall less whitefly population. Correlation analysis made between whitefly population, PDI and 
weather parameters showed that maximum temperature and minimum relative humidity was positively significant correlation with whitefly population in three locations Kavalur $(\mathrm{r}=0.079$ and $\mathrm{r}=0.673)$ (Table 2), Wadagnal $(\mathrm{r}=0.721$ and $\mathrm{r}=0.731)$ (Table 4) and Raichur $(\mathrm{r}=0.572$ and $\mathrm{r}=0.464)$ (Table 6). This indicates that rise in maximum temperature and low rainfall is conducive for whitefly population buildup. Whitefly population was negatively correlated but non-significant with minimum temperature in Kavalur and Raichur $(r=0.889$ and $r=0.684$ respectively). Similarly, Singh (1990) noted that hot weather with little or no rainfall was conducive for disease development of tomato leaf curl disease and also for multiplication of whitefly. Cooler weather with high relative humidity and rainfall were detrimental to whitefly population and spread. Board et al., (1993) found that population of whitefly and incidence of tomato leaf curl disease was high in August to October wherein rainfall will be very less with dry weather. Non-significant positive correlation of whitefly population with relative humidity observed in present study. These findings were in concurrence with the observations made by Srivastava and Prajapati (2012). Similarly in case of cotton, leaf curl virus, whitefly was found positively correlated with maximum temperature and relative humidity and negatively correlated with rainfall (Kadam et al., 2015).There was a similar finding noticed by Abhishek, et al.,
(2016), who confirmed the higher incidence of tomato leaf curl disease during October due to higher whitefly population. They also reported a positive correlation between whitefly population and maximum temperature and minimum temperature as observed in our results. Per cent disease incidence was negatively correlated with maximum temperature at Kavalur $(r=0.737)$, Wadagnal ( $\mathrm{r}=0.040)$ and Raichur ( $\mathrm{r}=0.707)$. The disease incidence showed positive relation with wind speed $r=0.674, r=0.654$ and $\mathrm{r}=0.218$ (non-significant) at Kavalur, Wadagnal and Raichur respectively which was similar as reported by Khan et al., (2012) and Majeed et al., (2016). Whereas, per cent disease incidence and whitefly population was positively correlated with each other in all the locations. Similar relation of whitefly and disease incidence was reported by Srivastava and Prajapati, 2012. To estimate the cumulative effects of different weather parameters and white fly population on disease outbreak regression analysis was carried out. Ali et al., (2015) observed that maximum temperature had negative correlation with YMD incidence.

Similarly Gupta and Varma (2015) reported that, whitefly population positively correlated with maximum temperature and nonsignificant positively correlated with relative humidity, our findings at Kavalur and Raichur are in line with these observations.

\section{GPS positions of selected plots for surveillance of MYMV incidence and its vector} Population

\begin{tabular}{|c|l|l|l|l|l|}
\hline Sl. No & \multicolumn{1}{|c|}{ Location } & District & Taluka & Latitude & Longitude \\
\hline 1. & Kavalur & Koppal & Koppal & 15.3355641 & 75.9196543 \\
\hline 2. & Wadagnal & Koppal & Koppal & 15.3353694 & 75.9178290 \\
\hline 3. & Raichur (MARS, UASR) & Raichur & Raichur & 16.1157404 & 77.1935041 \\
\hline
\end{tabular}

\begin{tabular}{|c|c|}
\hline Surveillance locations & Multiple regression equations \\
\hline Kavalur & $\mathrm{Y}=49.352+1.111 \mathrm{X} 1+0.339 \mathrm{X} 2-10.408 \mathrm{X} 3+15.733 \mathrm{X} 4+4.515 \mathrm{X} 5-8.721 \mathrm{X} 6+20.163 \mathrm{X} 7$ \\
\hline Raichur & $\mathrm{Y}=-516.486+0.125 \mathrm{X} 1-0.024 \mathrm{X} 2-50.125 \mathrm{X} 3+53.975 \mathrm{X} 4+11.594 \mathrm{X} 5-7.991 \mathrm{X} 6+22.303 \mathrm{X} 7$ \\
\hline Wadagnal & $\mathrm{Y}=176.387-0.210 \mathrm{X} 1-0.418 \mathrm{X} 2-5.238 \mathrm{X} 3-1.846 \mathrm{X} 4+0.205 \mathrm{X} 5-0.140 \mathrm{X} 6+2.121 \mathrm{X} 7$ \\
\hline
\end{tabular}


Table.1 Surveillance for whitefly population, MYMV incidence and weather parameters at Kavalur during 2016

\begin{tabular}{|c|c|c|c|c|c|c|c|c|c|c|}
\hline $\begin{array}{l}\text { Sl. } \\
\text { No. }\end{array}$ & $\begin{array}{c}\text { Duration of week } \\
(2016)\end{array}$ & $\begin{array}{c}\text { Standard } \\
\text { Meteorological } \\
\text { Week (SMW) }\end{array}$ & $\begin{array}{c}\text { Whitefly } \\
\text { population } \\
\text { /trap }\end{array}$ & PDI (\%) & $\begin{array}{c}\text { Rainfall } \\
(\mathbf{m m})\end{array}$ & $\begin{array}{l}\text { Min. } \\
\text { Temp } \\
\left(\begin{array}{l}\left.{ }^{0} \mathrm{C}\right) \\
\end{array}\right.\end{array}$ & $\begin{array}{l}\text { Max. } \\
\text { Temp } \\
\left({ }^{0} \mathrm{C}\right)\end{array}$ & $\begin{array}{c}\text { Min. } \\
\text { Humidity } \\
(\%)\end{array}$ & $\begin{array}{c}\text { Max. } \\
\text { Humidity } \\
(\%)\end{array}$ & $\begin{array}{c}\text { Max. Wind } \\
\text { speed }(\mathrm{km} / \mathrm{h})\end{array}$ \\
\hline 1 & June11- 17 & 24 & 26 & 0.00 & 15.7 & 22.99 & 32.84 & 47.33 & 89.09 & 2.47 \\
\hline 2 & June 18- 24 & 25 & 66 & 0.00 & 33.7 & 22.31 & 30.84 & 57.07 & 95.27 & 2.07 \\
\hline 3 & June 25- July 01 & 26 & 87 & 2.00 & 23.8 & 22.59 & 29.71 & 61.40 & 93.84 & 2.29 \\
\hline 4 & July 02- 08 & 27 & 85 & 8.00 & 11.1 & 22.99 & 29.79 & 62.49 & 95.46 & 2.63 \\
\hline 5 & July 09- 15 & 28 & 86 & 10.00 & 4.8 & 22.80 & 30.31 & 55.23 & 90.11 & 2.16 \\
\hline 6 & July $16-22$ & 29 & 63 & 15.00 & 3.3 & 21.73 & 29.61 & 58.33 & 93.31 & 2.06 \\
\hline 7 & July 23-29 & 30 & 81 & 25.00 & 41.3 & 21.47 & 29.61 & 62.09 & 99.29 & 2.57 \\
\hline 8 & July 30-Aug 05 & 31 & 56 & 29.00 & 5 & 22.37 & 28.50 & 63.34 & 93.70 & 2.50 \\
\hline 9 & Aug 06-12 & 32 & 72 & 32.00 & 1.3 & 22.61 & 30.61 & 55.37 & 89.99 & 2.89 \\
\hline 10 & Aug 13- 19 & 33 & 53 & 41.00 & 2.5 & 21.84 & 31.93 & 49.03 & 94.34 & 2.79 \\
\hline
\end{tabular}

Table.2 Correlation coefficients between MYMV incidence, whitefly population and weather parameters of Kavalur

\begin{tabular}{|c|c|c|c|c|c|c|c|c|}
\hline \multirow[t]{2}{*}{ Pearson correlation } & \multicolumn{8}{|c|}{ Correlations } \\
\hline & $\begin{array}{c}\text { Whitefly } \\
\text { population }\end{array}$ & PDI (\%) & Rainfall (mm) & Min. Temp $\left({ }^{0} \mathrm{C}\right)$ & $\begin{array}{c}\text { Max. Temp } \\
\left({ }^{0} \mathrm{C}\right)\end{array}$ & $\begin{array}{l}\text { Min. Humidity } \\
(\%)\end{array}$ & $\begin{array}{c}\text { Max. Humidity } \\
(\%)\end{array}$ & $\begin{array}{c}\text { Max.Wind speed } \\
(\mathrm{km} / \mathrm{hr})\end{array}$ \\
\hline Whitefly population & 1 & & & & & & & \\
\hline PDI (\%) & -.059 & 1 & & & & & & \\
\hline Rainfall (mm) & .204 & -.401 & 1 & & & & & \\
\hline Min. Temp $\left({ }^{0} \mathrm{C}\right)$ & $-.889^{* *}$ & .031 & .147 & 1 & & & & \\
\hline Max. Temp $\left({ }^{0} \mathrm{C}\right)$ & $.079^{*}$ & $-.737^{*}$ & .563 & .162 & 1 & & & \\
\hline Min. Humidity (\%) & $.673^{*}$ & -.054 & .292 & $.675^{*}$ & -.140 & 1 & & \\
\hline Max. Humidity (\%) & .391 & .153 & $.637^{*}$ & .332 & -.063 & .589 & 1 & \\
\hline Max.Wind speed (km/hr) & -.114 & $.674^{*}$ & -.251 & -.071 & -.340 & -.166 & .005 & 1 \\
\hline \multicolumn{9}{|c|}{ **. Correlation is significant at the 0.01 level (2-tailed). } \\
\hline Correlation is significant & ne 0.05 leve & ailed). & & & & & & \\
\hline
\end{tabular}


Table.3 Surveillance for whitefly population, MYMV incidence and weather parameters at Wadagnal during 2016

\begin{tabular}{|c|c|c|c|c|c|c|c|c|c|c|}
\hline Sl. No. & $\begin{array}{c}\text { Duration of week } \\
(\mathbf{2 0 1 6})\end{array}$ & $\begin{array}{c}\text { Standard } \\
\text { Meteorological } \\
\text { Week (SMW) }\end{array}$ & $\begin{array}{c}\text { Whitefly } \\
\text { population/trap }\end{array}$ & $\begin{array}{l}\text { PDI } \\
(\%)\end{array}$ & $\begin{array}{c}\text { Rainfall } \\
(\mathbf{m m})\end{array}$ & $\begin{array}{c}\text { Min. Temp } \\
\left({ }^{0} \mathrm{C}\right)\end{array}$ & 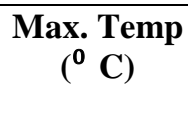 & $\begin{array}{c}\text { Min. } \\
\text { Humidity } \\
(\%)\end{array}$ & $\begin{array}{c}\text { Max. } \\
\text { Humidity } \\
(\%)\end{array}$ & $\begin{array}{c}\text { Max.Wind } \\
\text { speed }(\mathrm{km} / \mathrm{h})\end{array}$ \\
\hline 1 & June11- 17 & 24 & 0 & 0 & 7.5 & 23.54 & 32.23 & 46.29 & 81.06 & 5.60 \\
\hline 2 & June 18- 24 & 25 & 18 & 0 & 17.5 & 22.87 & 31.84 & 47.23 & 72.06 & 6.29 \\
\hline 3 & June 25- July 01 & 26 & 3 & 2 & 23.5 & 23.03 & 29.73 & 31.94 & 56.90 & 6.39 \\
\hline 4 & July 02- 08 & 27 & 22 & 3 & 12.5 & 23.56 & 29.94 & 43.39 & 73.47 & 7.23 \\
\hline 5 & July 09- 15 & 28 & 41 & 5 & 2 & 23.06 & 29.69 & 55.47 & 83.13 & 7.37 \\
\hline 6 & July 16-22 & 29 & 43 & 6 & 3 & 21.86 & 30.29 & 54.86 & 88.57 & 5.04 \\
\hline 7 & July 23-29 & 30 & 36 & 10 & 10.5 & 21.60 & 29.84 & 58.26 & 91.77 & 7.51 \\
\hline 8 & July 30-Aug 05 & 31 & 32 & 14 & 14.5 & 21.83 & 27.41 & 65.81 & 88.73 & 6.61 \\
\hline 9 & Aug 06-12 & 32 & 28 & 15 & 4 & 22.31 & 30.13 & 55.76 & 87.66 & 8.46 \\
\hline 10 & Aug 13- 19 & 33 & 16 & 15 & 5 & 21.84 & 30.86 & 52.11 & 91.27 & 8.41 \\
\hline
\end{tabular}

Table.4 Correlation coefficients between MYMV incidence, whitefly population and weather parameters of Wadagnal

\begin{tabular}{|c|c|c|c|c|c|c|c|c|}
\hline \multirow[t]{2}{*}{ Pearson correlation } & \multicolumn{8}{|c|}{ Correlations } \\
\hline & $\begin{array}{c}\text { Whitefly } \\
\text { population }\end{array}$ & PDI (\%) & Rainfall (mm) & Min. Temp $\left({ }^{0} \mathrm{C}\right)$ & Max. Temp $\left({ }^{0} \mathrm{C}\right)$ & $\begin{array}{l}\text { Min. Humidity } \\
(\%)\end{array}$ & $\begin{array}{c}\text { Max. Humidity } \\
(\%)\end{array}$ & $\begin{array}{c}\text { Max.Wind } \\
\text { speed }(\mathbf{k m} / \mathbf{h r})\end{array}$ \\
\hline Whitefly population & 1 & & & & & & & \\
\hline PDI (\%) & .407 & 1 & & & & & & \\
\hline Rainfall (mm) & -.514 & -.377 & 1 & & & & & \\
\hline Min. Temp $\left({ }^{0} \mathrm{C}\right)$ & .573 & .197 & -.367 & 1 & & & & \\
\hline Max. Temp ( $\left.{ }^{0} \mathrm{C}\right)$ & $.721^{*}$ & -.040 & -.197 & .063 & 1 & & & \\
\hline Min. Humidity (\%) & $.731^{*}$ & $.676^{*}$ & -.539 & .233 & .471 & 1 & & \\
\hline Max. Humidity (\%) & .600 & $-.701^{*}$ & $-.760^{*}$ & .161 & .363 & $.884^{* *}$ & 1 & \\
\hline Max.Wind speed $(\mathrm{km} / \mathrm{hr})$ & .111 & $.654^{*}$ & -.226 & .326 & -.301 & .210 & .290 & 1 \\
\hline
\end{tabular}


Table.5 Surveillance for whitefly population, MYMV incidence and weather parameters at Raichur during 2016

\begin{tabular}{|c|c|c|c|c|c|c|c|c|c|c|}
\hline Sl. No. & $\begin{array}{c}\text { Duration of week } \\
(2016)\end{array}$ & $\begin{array}{c}\text { Standard } \\
\text { Meteorological } \\
\text { Week (SMW) }\end{array}$ & $\begin{array}{c}\text { Whitefly } \\
\text { population/trap }\end{array}$ & $\begin{array}{l}\text { PDI } \\
(\%)\end{array}$ & $\begin{array}{l}\text { Rainfall } \\
(\mathbf{m m})\end{array}$ & $\begin{array}{l}\text { Min. } \\
\text { Temp } \\
\left({ }^{0} \mathrm{C}\right)\end{array}$ & $\begin{array}{l}\text { Max. } \\
\text { Temp } \\
\left({ }^{0} \mathrm{C}\right)\end{array}$ & $\begin{array}{c}\text { Min. } \\
\text { Humidity } \\
(\%)\end{array}$ & $\begin{array}{c}\text { Max. } \\
\text { Humidity } \\
(\%)\end{array}$ & $\begin{array}{c}\text { Max.Wind } \\
\text { speed }(\mathrm{km} / \mathrm{h})\end{array}$ \\
\hline 1 & June 04-10 & 23 & 295 & 0 & 54.5 & 23.89 & 33.73 & 49.37 & 95.86 & 2.30 \\
\hline 2 & June11- 17 & 24 & 247 & 0 & 4.0 & 24.57 & 33.79 & 45.40 & 87.51 & 3.04 \\
\hline 3 & June 18- 24 & 25 & 32 & 0 & 148.0 & 25.16 & 33.20 & 62.26 & 98.97 & 2.40 \\
\hline 4 & June 25- July 01 & 26 & 35 & 5.75 & 17.5 & 26.24 & 33.24 & 63.91 & 96.11 & 2.96 \\
\hline 5 & July 02- 08 & 27 & 161 & 10 & 3.0 & 26.93 & 34.37 & 58.97 & 94.56 & 3.46 \\
\hline 6 & July 09- 15 & 28 & 152 & 18 & 4.0 & 26.47 & 33.31 & 59.21 & 92.61 & 4.26 \\
\hline 7 & July $16-22$ & 29 & 200 & 20 & 38.5 & 26.00 & 34.01 & 59.51 & 94.81 & 1.99 \\
\hline 8 & July 23-29 & 30 & 52 & 26 & 140.5 & 25.37 & 32.40 & 71.13 & 99.90 & 1.66 \\
\hline 9 & July 30-Aug 05 & 31 & 54 & 32 & 60.5 & 25.47 & 30.91 & 75.50 & 99.10 & 3.07 \\
\hline 10 & Aug 06-12 & 32 & 89 & 38 & 0.5 & 25.73 & 34.64 & 58.29 & 99.56 & 3.70 \\
\hline
\end{tabular}

Table.6 Correlation coefficients between MYMV incidence, whitefly population and weather parameters of MARS, UAS, Raichur

\begin{tabular}{|c|c|c|c|c|c|c|c|c|}
\hline \multirow[b]{2}{*}{ Pearson correlation } & \multicolumn{8}{|c|}{ Correlations } \\
\hline & $\begin{array}{c}\text { Whitefly } \\
\text { population }\end{array}$ & PDI (\%) & Rainfall (mm) & Min. Temp $\left({ }^{0} \mathrm{C}\right)$ & Max. Temp $\left({ }^{0} \mathrm{C}\right)$ & $\begin{array}{l}\text { Min. Humidity } \\
(\%)\end{array}$ & $\begin{array}{c}\text { Max. Humidity } \\
(\%)\end{array}$ & $\begin{array}{c}\text { Max.Wind } \\
\text { speed }(\mathbf{k m} / \mathbf{h r})\end{array}$ \\
\hline Whitefly population & 1 & & & & & & & \\
\hline PDI (\%) & $.790^{* *}$ & 1 & & & & & & \\
\hline Rainfall (mm) & -.146 & .001 & 1 & & & & & \\
\hline Min. Temp $\left({ }^{0} \mathrm{C}\right)$ & $-.684^{*}$ & $-.864^{* *}$ & -.338 & 1 & & & & \\
\hline Max. Temp $\left({ }^{0} \mathrm{C}\right)$ & .572 & $-.707^{*}$ & -.227 & $.779^{* *}$ & 1 & & & \\
\hline Min. Humidity (\%) & .464 & .612 & .288 & $-.734^{*}$ & $-.962^{* *}$ & 1 & & \\
\hline Max. Humidity (\%) & .391 & .625 & .603 & $-.864^{* *}$ & -.564 & .616 & 1 & \\
\hline Max.Wind speed $(\mathbf{k m} / \mathbf{h r})$ & .239 & .218 & $-.644^{*}$ & .103 & -.109 & .137 & -.382 & 1 \\
\hline \multicolumn{9}{|c|}{ **. Correlation is significant at the 0.01 level (2-tailed). } \\
\hline Correlation is significant a & e 0.05 leve & & & & & & & \\
\hline
\end{tabular}


Table.7 Regression analysis of whitefly population, per cent disease incidence and weather parameters of MARS, UAS, Raichur during surveillance of MYMV

\begin{tabular}{|c|c|c|c|c|c|}
\hline \multicolumn{6}{|c|}{ ANOVA $^{b}$} \\
\hline Model & Sum of Squares & df & Mean Square & $\mathrm{F}$ & Sig. \\
\hline Regression & 1719.398 & 7 & 245.628 & 12.545 & $.076^{\mathrm{a}}$ \\
\hline Residual & 39.159 & 2 & 19.579 & & \\
\hline $\mathrm{R}$ & $.989^{\mathrm{a}}$ & & & & \\
\hline R square & .978 & & & & \\
\hline Multiple regression equation & \multicolumn{5}{|c|}{$Y=-516.486+0.125 X 1-0.024 X 2-50.125 X 3+53.975 X 4+11.594 \times 5-7.991 \times 6+22.303 X 7$} \\
\hline \multicolumn{6}{|c|}{$\begin{array}{l}\text { a. Predictors: (Constant), Wind speed, Maximum temperature, Rainfall, Sunshine hours, Maximum relative humidity, Minimum temperature, } \\
\text { Minimum relative humidity }\end{array}$} \\
\hline \multicolumn{6}{|l|}{ b. Dependent Variable: WFP } \\
\hline
\end{tabular}

Table.8 Regression analysis of whitefly population, per cent disease incidence and weather parameters of Kavalur during surveillance of MYMV

\begin{tabular}{|c|c|c|c|c|c|}
\hline \multicolumn{6}{|c|}{ ANOVA $^{b}$} \\
\hline Model & Sum of Squares & df & Mean Square & $\mathrm{F}$ & Sig. \\
\hline Regression & 1905.910 & 7 & 272.273 & 16.164 & $.059^{\mathrm{a}}$ \\
\hline Residual & 33.690 & 2 & 16.845 & & \\
\hline $\mathrm{R}$ & $.991^{\mathrm{a}}$ & & & & \\
\hline R square & .983 & & & & \\
\hline Multiple regression equation & \multicolumn{5}{|c|}{$Y=899.040+0.037 X 1+0.017 X 2-4.571 X 3-23.563 X 4-5.544 X 5+1.710 \times 6+36.719 X 7$} \\
\hline \multicolumn{6}{|c|}{$\begin{array}{l}\text { a. Predictors: (Constant), Wind speed, Maximum temperature, Rainfall, Sunshine hours, Maximum relative humidity, Minimum temperature } \\
\text { Minimum relative humidity }\end{array}$} \\
\hline \multicolumn{6}{|l|}{ b. Dependent Variable: WFP } \\
\hline
\end{tabular}


Table.9 Regression analysis of whitefly population, per cent disease incidence and weather parameters of Wadaganal during surveillance of MYMV

\begin{tabular}{|c|c|c|c|c|c|}
\hline \multicolumn{6}{|c|}{ ANOVA $^{b}$} \\
\hline Model & Sum of Squares & $\mathrm{df}$ & Mean Square & $\mathrm{F}$ & Sig. \\
\hline Regression & 320.214 & 7 & 45.745 & 9.349 & $.100^{\mathrm{a}}$ \\
\hline Residual & 9.786 & 2 & 4.893 & & \\
\hline $\mathrm{R}$ & $.985^{\mathrm{a}}$ & & & & \\
\hline R square & .970 & & & & \\
\hline Multiple regression equation & \multicolumn{5}{|c|}{$Y=176.387-0.210 \times 1-0.418 \times 2-5.238 \times 3-1.846 \times 4+0.205 \times 5-0.140 \times 6+2.121 \times 7$} \\
\hline \multicolumn{6}{|c|}{$\begin{array}{l}\text { a. Predictors: (Constant), Wind speed, Maximum temperature, Rainfall, Sunshine hours, Maximum relative humidity, Minimum tempera } \\
\text { Minimum relative humidity }\end{array}$} \\
\hline b. Dependent Variable: WFP & & & & & \\
\hline
\end{tabular}

X1- Whitefly population X2- Rainfall $(\mathrm{mm}) \mathrm{X} 3$ - Minimum temperature $\left({ }^{0} \mathrm{C}\right) \mathrm{X} 4$ - Maximum temperature $\left({ }^{0} \mathrm{C}\right)$

X5- Minimum relative humidity (\%) X6-Maximum relative humidity (\%) X7-Windspeed (km/hr) 
Fig.1 Weekly observation on MYMV incidence in mungbean at Kavalur

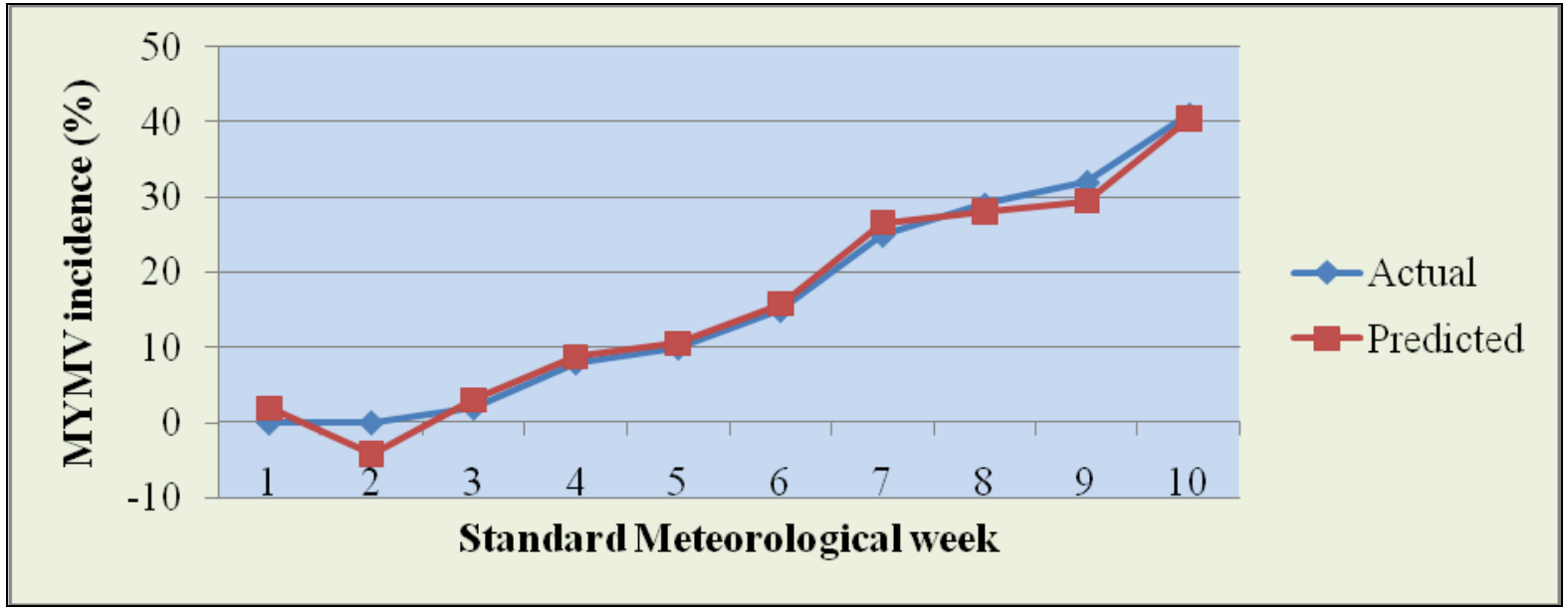

Fig.2 Weekly observation on MYMV incidence in mungbean at Wadagnal

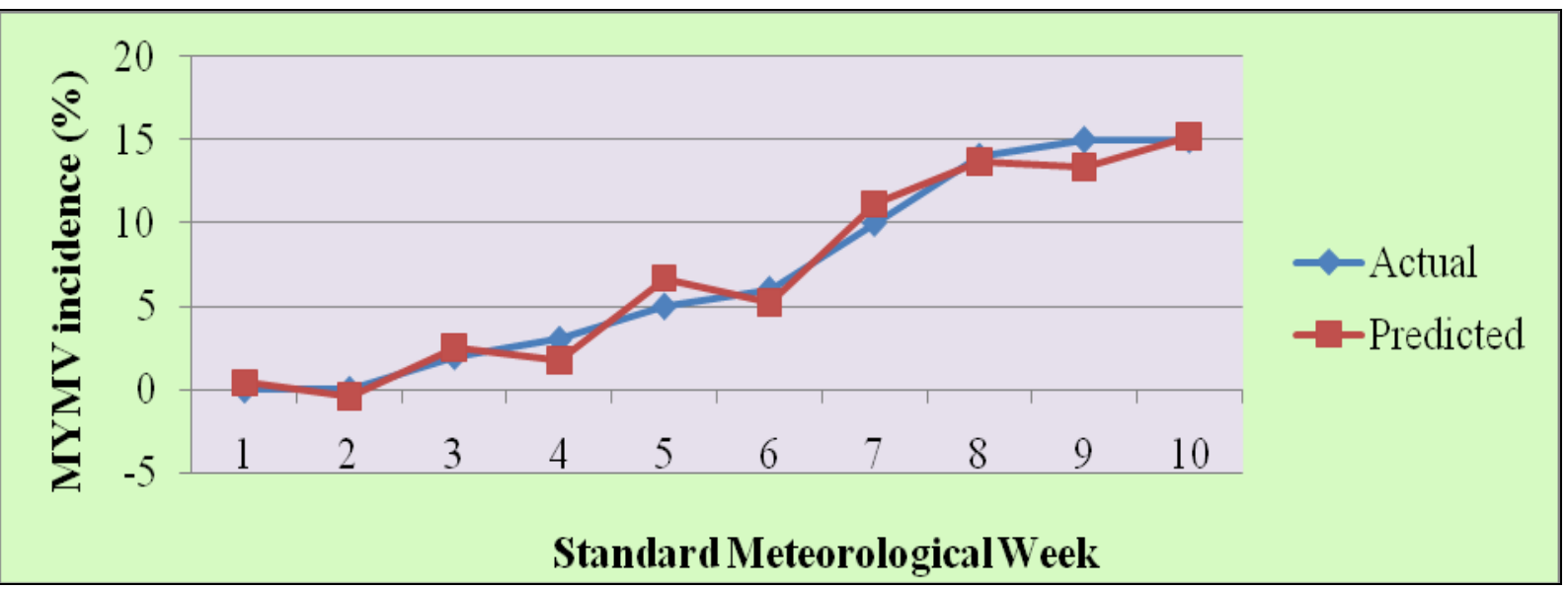

Fig.3 Weekly observation on MYMV incidence in mungbean at UAS, Raichur

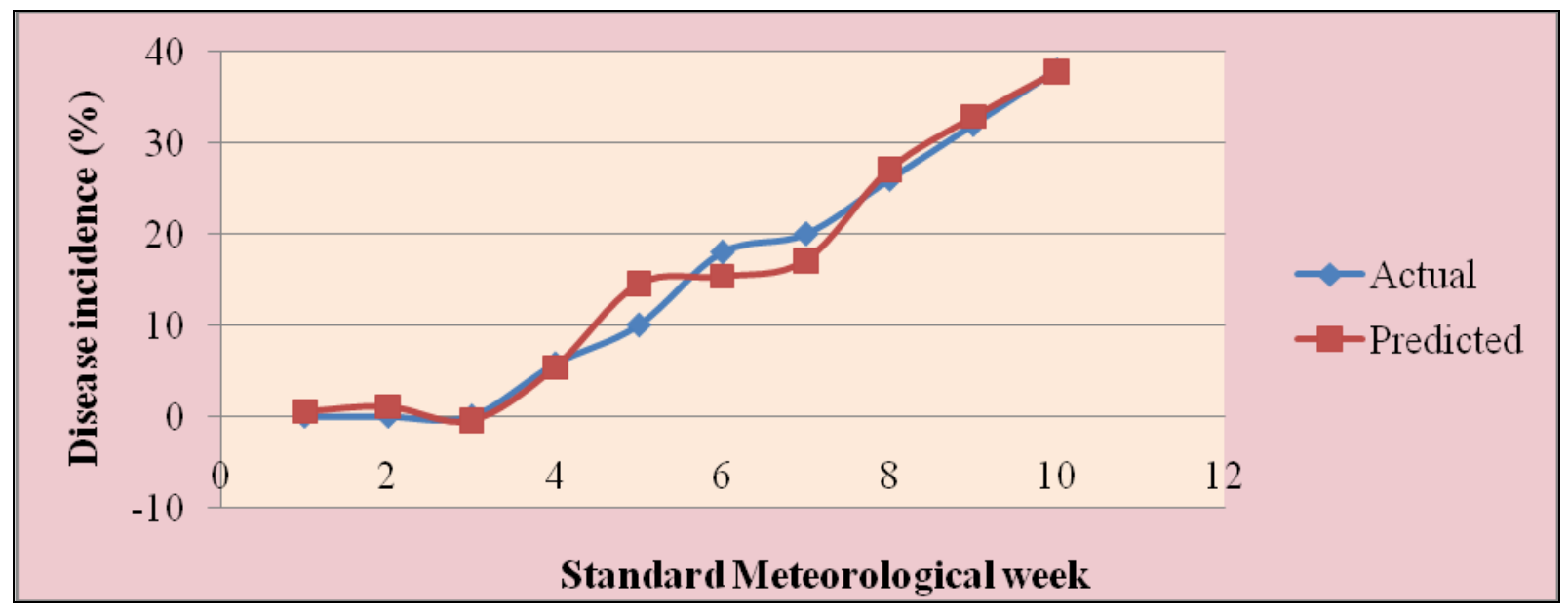


The regression analysis revealed that the contribution or influence of weather parameters on whitefly population and disease incidence was of about 97.80 per cent (Fig. 1) $\left(\mathrm{R}^{2}=0.978\right)$ at Kavalur (Table 7), 98.30 per cent (Fig. 2) $\left(\mathrm{R}^{2}=0.983\right)$ at Wadagnal (Table 8) and 97.00 per cent (Fig. 3) $\left(\mathrm{R}^{2}=0.97\right)$ at Raichur (Table 9). Further which are plotted in figures based on differences between actual and predicted values. Following are the equations obtained after multiple regression analysis of data from all the three locations individually for prediction of yellow mosaic disease incidence in surveillance plots.

The above simple rules may be utilized in formulation of bi-weekly district level agromet advisory bulletins and also by extension workers to make tactical decisions for MYMV control measures.

Surveillance studies in all three locations revealed that, weather parameter like maximum temperature had positive significant correlation with whitefly population and disease incidence has correlation with maximum relative humidity and maximum temperature and which intern indicates that higher the maximum temperature more is whitefly population and increase in relative humidity increase in disease. In addition, it was also evident that prediction models of yellow mosaic disease vary from location to location based on the cropping pattern and weather patterns. However, the correlation between whitefly population and maximum temperature was found significantly positive in most of the cases as previously reported.

From the present study it was concluded that out of five weather variables, only minimum temperature and rainfall had statistically significant correlation with MYMV outbreak. Rise in maximum temperature was conducive for development of disease; while increase in relative humidity and heavy rainfall was detrimental to whitefly population. These findings can be used to develop a disease forecasting model for judicious application of chemicals. Long term data on disease incidence should be utilized for refinement of the predictive model and its validation under difference temperature and moisture regimes before putting it into operational use.

\section{References}

Abhishek, S. K., Singh and Shiwangi, 2016. Studies on effect of weather conditions on whitefly (Bemisia tabaci) population and tomato leaf curl disease. Res. Environ. Life Sci., 9(4): 470-472.

Ali, S., Khan A., Zeshan A., Habib, A. and Haider, M. S., 2015. Characterization of conducive environmental conditions for mungbean yellow mosaic virus disease incidence on mungbean germplasm. Pak. J. Phytopathol., 27 (01): 27-30.

Borad, V. K., Puri, S. N., Brown, J. K. and Butler, G. D., 1993. Relationship of Bemisia tabaci population density and tomato leaf curl disease incidence in tomato. Pest Mangt. Eco. Zool., 1: 1419.

Gupta, K. N., and Verma, R. K., 2015. Ecological traits of yellow mosaic disease in relation to epidemics in soybean. JNKVV Res. J., 49 (2): 256261.

Haq, Q. M. I., Arif, A. and Malathi V. G., 2011. Infectivity analysis of a blackgram isolate of mungbean yellow mosaic virus and genetic assortment with MYMIV in selective hosts. Virus Res., 42 (3):429-439.

Hull, R., 2004. Mathew's Plant Virology. Fourth Edition. Elsevier Publishers, India, pp.180-182.

Kadam, D. B., Kadam, D. R. and Umate, S. M. 2015. Effect of weather parameters on incidence of sucking pests on Bt. 
Cotton. Int. J. Plt. protec., 8(1): 211213.

Khan, M. A., Rashid, A. and Mateen, A. 2012. Incidence of mungbean yellow mosaic virus, its epidemiology and management through Mycotal, Imidachloprid and Tracer. Agri. and Bio. J. N. America., 3(11): 476-480.

Majeed, M. Z., Mudassar Javed, Muhammad Asam Riaz and Muhammad Afzal. 2016. Population Dynamics of Sucking Pest Complex on Some Advanced Genotypes of Cotton under Unsprayed Conditions. Pak. J. Zool., 48(2): 475480.

Meena, R.S., Rathore, G.S., Shekhawat, B.S.,
Yadav, L.D. and Agnihotri, J.P. 2013. Population dynamics of sucking pests and their correlation with weather paramaters in chilli (Capsicum annum). Ind. J. Mycol. Pl. Pathol. 14(3): 304309.

Singh, S. J., 1990. Etiology and epidemiology of whitefly-transmitted virus diseases of tomato in India. Plant Dis. Res., 5: 6470 .

Srivastava, A. K., and Prajapati, R. K. 2012. Influence of weather Parameters on Outbreak of Mungbean Yellow Mosaic Virus in Black Gram of Bundelkhand Zone of Central India. J. Agric. Physiol., 12(2): 143-151.

\section{How to cite this article:}

Meghashree Meti, Mallikarjun Kenganal, D.S. Ashwathnarayana and Gururaj Sunkad. 2017. Surveillance of Mungbean Yellow Mosaic Virus (MYMV) Incidence and Its Vector Population for Development of Yellow Mosaic Disease Forewarning. Int.J.Curr.Microbiol.App.Sci. 6(10): 94-104. doi: https://doi.org/10.20546/ijcmas.2017.610.011 